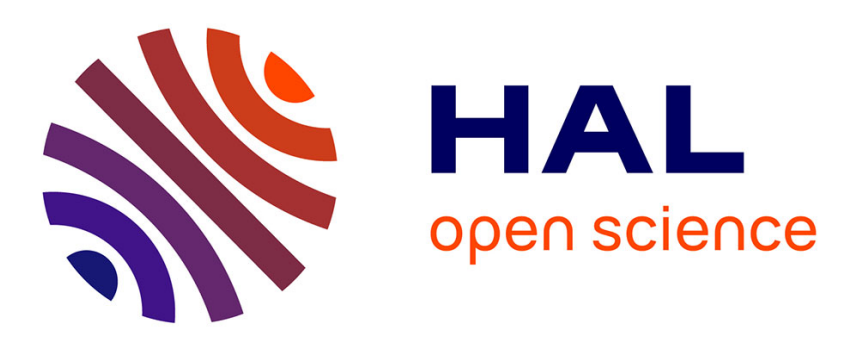

\title{
Dynamic Behavior of an Interactive XY -6 DoF Simulator for People with Reduced Mobility
}

Taha Houda, Lotfi Beji, Ali Amouri, Malik Mallem

\section{To cite this version:}

Taha Houda, Lotfi Beji, Ali Amouri, Malik Mallem. Dynamic Behavior of an Interactive XY -6 DoF Simulator for People with Reduced Mobility. 4th IEEE Conference on Control Technology and Applications (CCTA 2020), Aug 2020, Montreal, Canada. pp.522-527, 10.1109/CCTA41146.2020.9206265 . hal-02879923

\section{HAL Id: hal-02879923 https://hal.science/hal-02879923}

Submitted on 24 Jun 2020

HAL is a multi-disciplinary open access archive for the deposit and dissemination of scientific research documents, whether they are published or not. The documents may come from teaching and research institutions in France or abroad, or from public or private research centers.
L'archive ouverte pluridisciplinaire HAL, est destinée au dépôt et à la diffusion de documents scientifiques de niveau recherche, publiés ou non, émanant des établissements d'enseignement et de recherche français ou étrangers, des laboratoires publics ou privés. 


\title{
Dynamic Behavior of an Interactive XY - 6 DoF Simulator for People with Reduced Mobility
}

\author{
Taha Houda ${ }^{1}$, Lotfi Beji ${ }^{2}$, Ali Amouri ${ }^{3}$ and Malik Mallem ${ }^{4}$
}

\begin{abstract}
Sliding simulators for people with poor or reduced mobility, such as skiing, jet skiing, and wake, are new fields of motion simulator while all existing simulators for sliding movement, from our knowledge, use a low degree of freedom robot which limits the reproduction of the human sensations. In this paper, we present a high degree of freedom simulator with a hybrid structure composed of a parallel 6 DoF GoughStewart robot mounted on a serial XY table, we present the building of mixed reality environment, then, we propose two scenarios for operating the simulator: manual or interactive mode and automatic mode. At the interactive mode level, we present the way-points tracking and virtual obstacle avoidance and at the automatic mode level, we present the planning of predefined trajectory and the application of classic and adaptive approaches of motion cueing algorithm on the real platform.
\end{abstract}

\section{INTRODUCTION}

The loss of autonomy and mobility, for a large number of individuals around the world about $15 \%$ from the world population [1], is mainly due to road accident victims, people with disabling diseases like multiple sclerosis and of course elderly people living alone. A significant percentage of the population, around $10 \%$ [1], use wheelchairs.

The project goal is to offer a sensational alternative feeling, through the study and the production of an open platform, with a significant number of degrees of freedom. It has been proven that this type of device ensures psychological, muscular, and social benefits to people with severe disabilities [2]. With the integration of mixed reality, in which our team has great expertise [3], [4], on one hand, and coupled with a hybrid structure robot, on the other hand, we want to present a complete set to ensure that all the benefits of a total immersion be accomplished.

The Gough-Stewart platform has been widely used for simulator applications especially for driving and flight simulation due to its advantages like the high payload and low error compared with serial robots [5]. To overcome the known workspace limitation of the parallel robot, many hybrid structures, that combine serial and parallel architecture, have been proposed such as a parallel robot mounted on one axis [6] or two-axis [7]. These kinds of simulators can offer a better acceleration rendering; thus, a high immersive feeling. The objective of the project is to offer a large workspace sliding simulator for gaming and rehabilitation purposes. Our simulator called Evr@, which consists of the well known Gough-Stewart platform mounted on two sliding

\footnotetext{
1,2,3,4 Taha HOUDA, Lotfi BEJI, Ali Amouri, Malik Mallem, IBISC, Univ Evry, Université Paris-Saclay 91025 Evry, France. (Taha.houda, Lotfi.Beji, Ali.Amouri, Malik.Mallem) Quniv-evry.fr
}

joints, has been fully designed and developed from scratch by our research team IRA2 in the IBISC-Laboratory [8].

In fact, all motion simulators consist of three major parts: robotics, virtual reality environment, and humans (users). Our aim is to define the interactive way between the human mounted on the hybrid structure robot, and the virtual environment, thus the interaction can be done in two ways:

- Scenario 1: Manual or interactive one, it means that the human operator defines the way-points for both hybrid robot and virtual environment, in this case, the system is able to localize and recognize the human gestures thanks to a joystick. It is supposed that the joystick is calibrated with the virtual environment, thus, the virtual environment is able to detect any obstacles in the scene where we define a control law to track the way-point and avoid the detected obstacles (see section IV).

- Scenario 2: Automatic one, it means that the human has no influence on the virtual environment and it is assumed to be passive. The trajectory in the virtual environment is predefined, the objective of this scenario is to control the hybrid robot simulator to track the achievable predefined trajectory to generate, in the known limitations, an artificial sliding sensation for the human operator (see section VI).

In this paper, we present firstly, a brief description of Evr@ mechatronics. Then, we present the design of a mixed reality scene. After we detail a control law that ensures way-points tracking with virtual obstacle avoidance for scenario 1 and we present the trajectory planning of the ski-model in the virtual scene for scenario 2 with the generation of achievable predefined trajectory based on Motion Cueing Algorithm (MCA). The following figure shows the human integration on Evr@ simulator in an immersive environment.

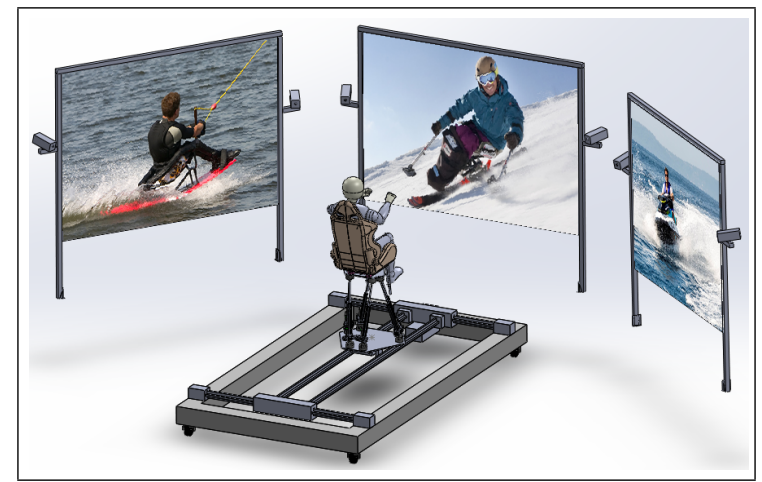

Fig. 1. Sliding simulator for ski, jet-ski and wake application 


\section{Mechatronics Description}

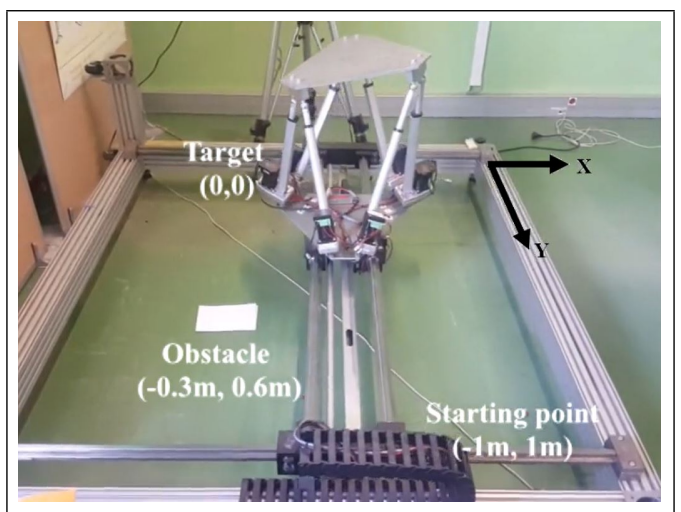

Fig. 2. Real figure of the XY - 6 DoF Mechatronics system

Fig.2 shows the multi-body structure, the well known Gough-Stewart platform mounted on two sliding joints. The 8 joints are actuated using a Nanotec PD4 servomotor where the rotational movement of the leg is transformed into translational movement due to the high-quality ball screwup system. Below, we present the global architecture of our mechatronics simulator with technological used parts.

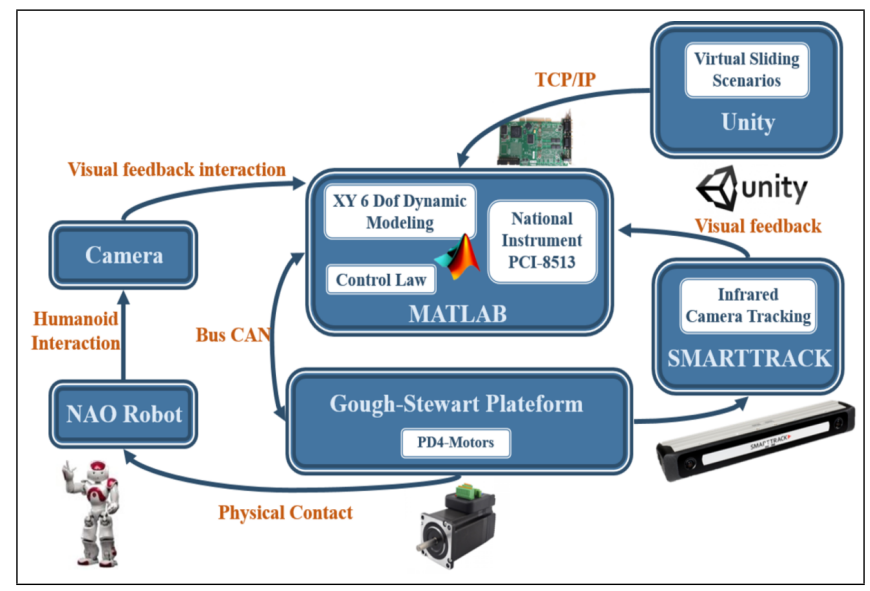

Fig. 3. Technological mechatronics architecture

A virtual sliding scenario should be designed under Unity software that should be simultaneously projected on a screen. The trajectory information is shared with the robotic structure via MATLAB through TCP/IP connection. The control instructions are transmitted by a CAN bus via the National Instrument PCI-8513 to motors that move the platform. Several types of sensors will be used to have better robot control, such as displacement, velocity, and torque sensors of PD4 motors as well as visual feedback from an infrared camera. Humanoid robot NAO takes place to replace the human for security reasons and for algorithm validation.

\section{MiXed REALITy SCENARIO DESIGN UNDER UNity}

In recent years, Handisports have evolved due to the increasing number of humans with poor or disabled mobility, especially the handicapped skier. In order to design a virtual scenario under Unity software meeting the criteria of human disabled sport, it is advisable to move towards references of places and models of equipment adapted to the practice. From these references, it is possible to generate a landform as well as an avatar model.

In this section, firstly, we develop an appropriate ski-model (Dual-Ski) used in real life, then we model an existing track trajectory and finally we model a real mountain to be as close as possible to the total immersive sensation and to build the most possible realistic environment. To choose the appropriate real ski frame, several options are available at the hardware level. Indeed, alpine skiing has several types

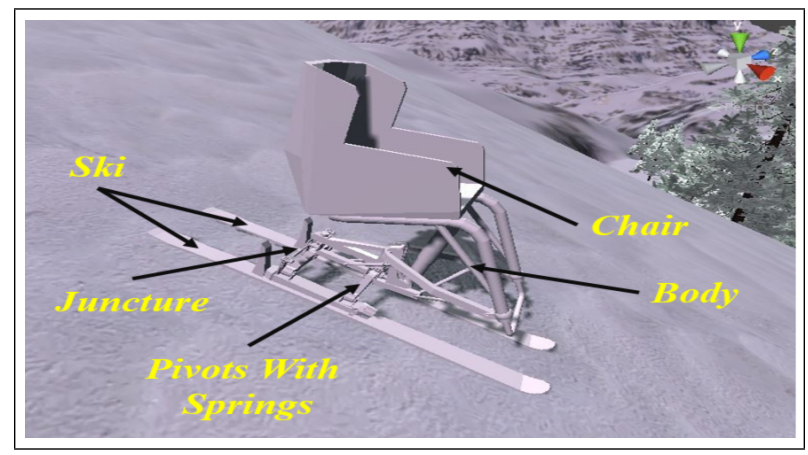

Fig. 4. Model of the ski frame for disabled person

of frames allowing different experiences we cite: Ski-Kart, Uni-Ski, and Dual-Ski [9]. The ski frame is designed to have the knee joint above the hip joint where the user has better cardiorespiratory responses [10]. The design of the ski frame model is done via the Blender software inspired by the model Dual-ski Loisir de Tessier that can provide the highest sensations compared with the others design and it is presented in Fig.4. The tracks choice was made on a track in the station of Combloux at Haute-Savoie (Northern

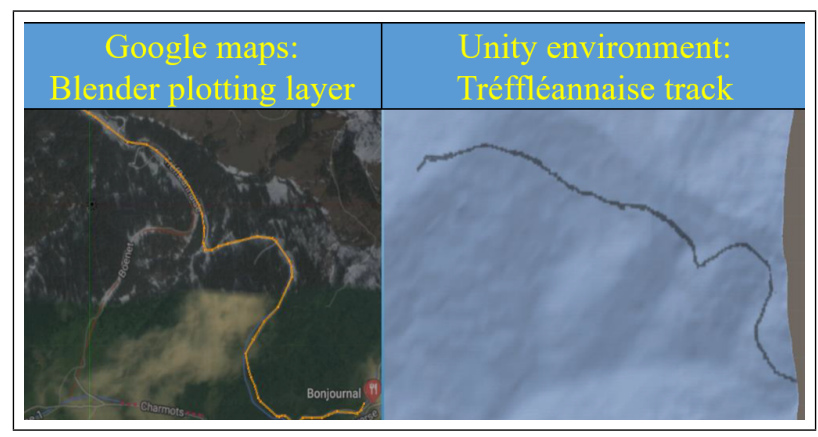

Fig. 5. Tréffléannaise track definition (top view)

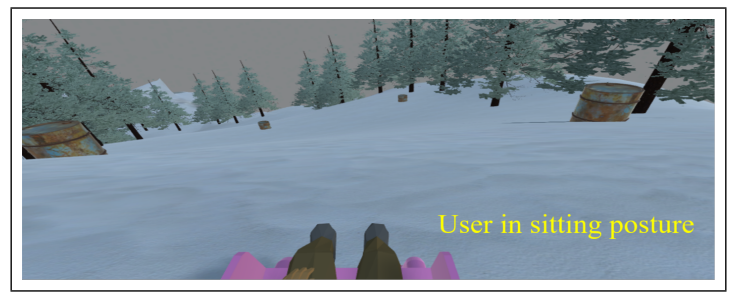

Fig. 6. User view of a disabled person in the virtual ski scene

Alps of France). The track is called Tréffléannaise, it starts at 
Bonjournal (1853m above sea level) and ends at La Giettaz (1200m above sea level). It meets the desired criteria since it is reserved for hand skis and is surrounded by trees along its entire length. The orange line on the left side of Fig. 5, represents the $3 \mathrm{D}$ modeling curve corresponding to the real track. Fig.6 shows the user view of the final environment. After assembling all parts, we had built a full mixed reality environment ready to be coupled with the robotics platform in both scenarios.

\section{First SCEnARIO: MANUAL OR InTERACTIVE MODE}

In previous work, the dynamic and energetic modeling of the Evr@ simulator was done in [11], which takes the following form [12].

$$
\Gamma={ }^{b} J_{p}^{T}\left({ }^{b} F_{p}+{ }^{b} F_{e}\right)+\sum_{i=1}^{6}\left(\frac{\partial \dot{q}_{i}}{\partial \dot{q}_{a}}\right)^{T} H_{i}(q, \dot{q}, \ddot{q})
$$

with, $\Gamma$ - vector of the actuator torques/forces, ${ }^{b} J_{p}$ - Jacobian matrix, ${ }^{b} F_{e}$ - external wrench (forces and moments) exerted on the platform, $\dot{q}_{i}$ - passive joints speed, $\dot{q}_{a}$ - active joints speed, $H_{i}(q, \dot{q}, \ddot{q})$ - is the inverse dynamic model of leg $i$ considering as a serial robot and different methods can be used to calculate this vector [13], ${ }^{b} F_{p}$ - the total forces, ${ }^{b} F_{p}$ is calculated by the following Newton-Euler equation:

$$
{ }^{b} F_{p}={ }^{b} \mathbf{I}_{p}{ }^{b} \dot{\mathbf{v}}_{p}+\left[\begin{array}{c}
{ }^{b} w_{p} \times\left({ }^{b} w_{p} \times{ }^{b} M S_{p}\right) \\
{ }^{b} w_{p} \times\left({ }^{b} I_{p}{ }^{b} w_{p}\right)
\end{array}\right]-\left[\begin{array}{c}
M_{p} I_{3} \\
{ }^{b} M \hat{S}_{p}
\end{array}\right]{ }^{b} g
$$

where, $M_{p},{ }^{b} I_{p}-(3 \times 3),{ }^{b} \mathbf{I}_{p}-(6 \times 6),{ }^{0} M S_{p}-(3 \times 1)$ are mass, inertia tensor, spatial inertia matrix and first moment of inertia vector of the platform, respectively.

The Evr@ simulator inertial and friction parameters are of number 210 that was optimized to 90 and identified in [11]. For this scenario, the Gough-Stewart platform plays the role of tracking the angular movement where the platform reaches the maximum angle when it is on the same horizontal line of the obstacle. This task requires fast motion and high dynamic accuracy, therefore, an application of the well known computed torque control presented in [13] was applied. In this section, we present control that ensures the tracking of waypoints given by the user while avoiding the virtual obstacles and it is applied only for the XY table.

\section{A. System and problem description}

In this work, we consider the platform as point mass described as a circle with center $\mathrm{q}$ and radius $\mathrm{r}$, the way-points are given by the user are $T_{i}$ and the virtual obstacles are $O_{i}$. Two control laws will be conducted in the following ensuring the stabilization and the regulation of robot kinematics and dynamics.

$$
\text { Kinematics: } \dot{q}=u \quad \text { (3a) or dynamics: } \ddot{q}=u
$$

\section{B. Obstacle localization of the moving platform}

Let us introduce, $O_{q^{-}}$the point of intersection between the circle and the line between $\mathrm{O}$ and $\mathrm{q}$, thus when the robot approaches an obstacle, the $O_{q}$ position approaches $O$.

$$
O_{q i}=q+r \frac{O_{i}-q}{\left\|O_{i}-q\right\|}
$$

where, $\|$.$\| is the Euclidean norm.$

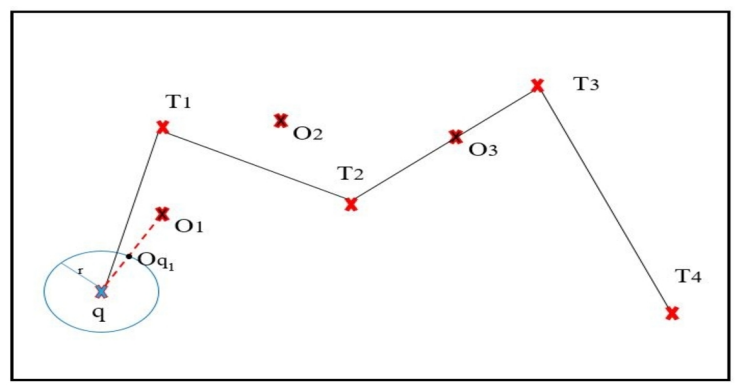

Fig. 7. Trajectory without collision avoidance

\section{Control Law Definition}

A dual-function feedback control law was developed, the law of control is the sum of an attractive term $U_{a}$ responsible for the robot's convergence towards the target and a repulsive term $U_{r}$ that allows it to avoid the obstacle. This is based on a conceptual approach that can be summarized as follows:

If $U_{a}$ is a control law that makes the robot converge to the desired location, then control law $u_{a}+u_{r}$, where $u_{r}$ is a function based on the function of Lyapunov and does not change with regard to the region where the robot is located and makes the system converge to the same required location. Using this function $U_{r}$, to avoid the contact between obstacles and a set of points around the robot, one could modify the trajectory behavior. The control law we are proposing also guarantees the robot's fluid behavior before the obstacle.

1) Stabilization and Regulation Equation of kinematics: Our main result is to extend the issue of stabilizing control to include a regulation function. Consequently, the regulation function is introduced in the following theorem.

Theorem 1: Let $A \subset \mathbb{R}^{n}$ be a set that contains the equilibrium. We consider $u_{a}(q)$ a stationary control that stabilize the system (3a) around an equilibrium point and $V$ : $\mathbb{R}^{n} \times[0,+\infty[\rightarrow \mathbb{R}$ the Lyapunov function associated with the function $u_{a}(q) \in \mathbb{R}^{m}$. Thus, for any function $\xi: \mathbb{R}^{n} \rightarrow \mathbb{R}$ continuous on $A$, the control law.

$$
u=u_{a}(q)+\xi\left(\frac{\partial V}{\partial q}\right)^{o r t h}
$$

Makes the system (3a) uniformly asymptotically stable at a target point. () ${ }^{\text {orth }}$ denote the orthogonal vector.

Proof: From theorem 1 the system (3a) is asymptotically stable by the control law $u_{a}$. Then according to Kurzweil's theorem, there exists a Lyapunov $V$ function associated with the system $\dot{q}=u_{a}$. This gives us that:

$$
\dot{V}=\frac{\partial V}{\partial q} u_{a}<0
$$

Using the same function of Lyapunov for the system (3a) associated with the control law (5), proposed in Theorem 1, we obtain:

$$
\dot{V}=\frac{\partial V}{\partial q} u=\frac{\partial V}{\partial q}\left[u_{a}+\xi\left(\frac{\partial V}{\partial q}\right)^{\text {orth }}\right]=\frac{\partial V}{\partial q} u_{a}<0
$$


Hence, according to the theorem of Lyapunov, the solutions of (3a) converge towards the desired position.

This theorem allows us to define the control law into the sum of two-part attractive and repulsive terms where $\xi$ is the regulation function vector. The changing of $\xi$ will change the trajectory behavior while preserving the system convergence.

2) Stabilization and Regulation Equation of dynamics:

For second-order control systems, the dual integrator has generally been used to transfer the dynamic problem into a kinematic problem, which is easier to handle given the number of theories applied to this type of system. The problem with the double integrator is that if one has a fully actuated dynamic system, it becomes underactuated, which means the number of states will be greater than the actuators and if it is used for an underactuated system, the degree of under actuation will deepen. Furthermore, the number of states of the system will be multiplied by two which complicates the search for a control law, therefore, the dynamic problem will be transformed to a kinematic problem while conserving the number of states and actuation based on the theorem presented in [14], [15] which is:

Theorem 2: Let define the following control equation:

$$
\ddot{e}=f(e, \dot{e}, u)
$$

Where $e$ is a scalar that represents the state and $u$ the control. If there is $u$ that asymptotically converts to 0 the solution of the following equation: $\dot{r}=f(e, \dot{e}, u)+\alpha \dot{e}$ with $r_{0}=\dot{e}_{0}+\alpha e_{0}$ where, $r \in L^{\infty} \cap L^{2}$, therefore, $e$ and $\dot{e}$ converge asymptotically to 0 .

\section{Regulation Function Definition}

For Kinematic case,

Theorem 3: let $q$ be a solution of the system (3a) resulting from $q_{0}\left(x_{0}, y_{0}\right), V=\frac{\|q\|^{2}}{2}$ and $L$ the function defined on $\mathbb{R}$ such that its representative curve is the line that passes through the target $\mathrm{T}$ and $\mathrm{O}$ respectively of the target and the obstacle.

$$
\xi=-\frac{\operatorname{sign}\left(\left[y_{0}-L\left(x_{0}\right)\right]\left[T_{x}-O_{x}\right]\right)}{\left\|O_{q}-O\right\|}
$$

The solution $q$ of presented $\xi$ verifies the following assertions: $O_{q}$ avoid $O$ and $q$ converges asymptotically to $T$

Proof: The relatively simple choice of the Lyapunov function

$$
V=\frac{\|q\|^{2}}{2}
$$

Proves the asymptotically stablility of the system (3a) at point $T(0,0)$ if one poses $u=u_{q}=-q$. By using the same Lyapunov Function, from theorme 2 the controller is of this form

$$
\dot{q}=-q+\xi(\nabla q)^{\text {orth }}=-q+\xi q^{\text {orth }}
$$

which ensures the asymptotic stability of the system (3a) at point $\mathrm{T}(0,0)$ for any scalar function of $\xi$. It is clear that $O_{q}$ avoids $O$ by simple $\lim _{O_{q} \rightarrow+O} \xi= \pm \infty$.

Therefore, the kinematic controller can be written as follow:

$$
\dot{q}=-\lambda \nabla V(q)+\xi(\nabla V(q))^{\text {orth }}
$$

where, $\lambda \in \mathbb{R}_{+}^{*}, V: \mathbb{R} \rightarrow \mathbb{R}_{+}$defined positive as $\forall q \neq 0$ we have $\nabla V(q) \neq 0$. The control associated with the dynamics using the same methodology and by taking the Lyapunov Function $V=\frac{\|N\|^{2}}{2}$, where $N=\dot{q}+\alpha q$ can be written as,

$$
u=-(\beta+\alpha) \dot{q}-\alpha \beta q+\xi(\dot{q}+\alpha q)^{\text {orth }}
$$

Where, $\beta$ is a chosen parameter, where,

$$
\xi=\frac{\operatorname{sign}\left(\left[\dot{y}_{0}+\alpha y_{0}-L\left(\dot{x}_{0}+\alpha x_{0}\right)\right]\left[T_{x}-O_{x}\right]\right)}{\left\|\dot{O}_{q}+\alpha O_{q}-O\right\|}
$$

Robustness and efficiency of the proposed controls are verified and simulated using SimMechanics (physical engine).

\section{CONTROL IMPLEMENTATION AND VALIDATION}

The simscape (SimMechanics) software provides a multibody simulation environment for a 3D mechanical system such as robots [16]. The Evr@ simulator was designed

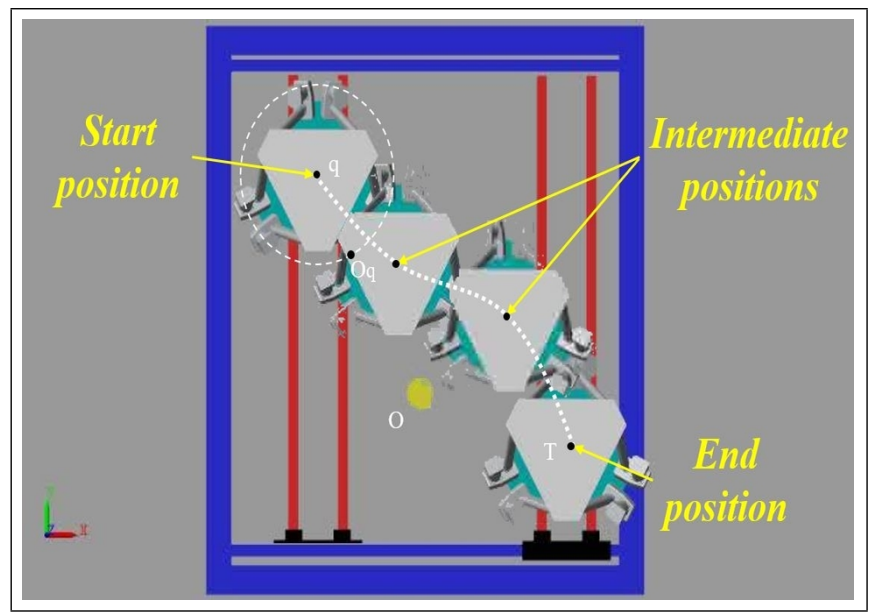

Fig. 8. Platform navigation to the right of the obstacle in SimMechanics

and modeled using a large number of blocks offered in SimMechanics for dynamic modeling presented in Fig. 8,

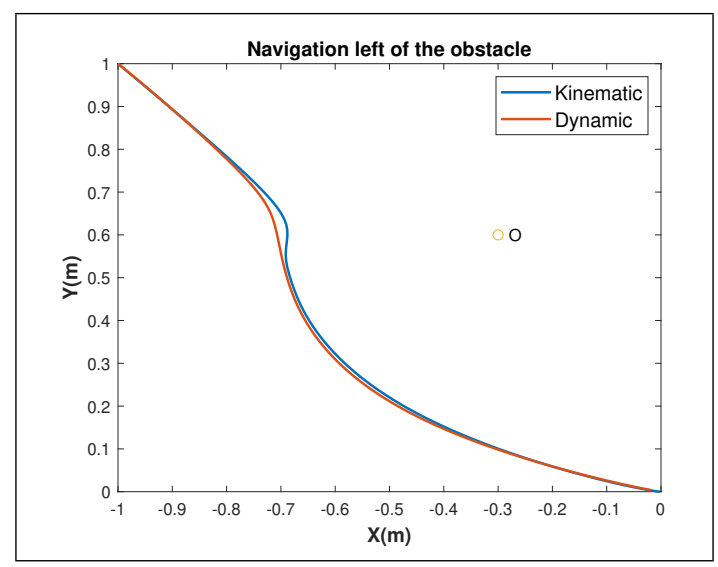

Fig. 9. Platform navigation to the left of the obstacle

where the control laws are applied. To simplify, only oneway point and one obstacle will be taken. At $t=0 \mathrm{~s}$, center of the platform is located at $(-1 \mathrm{~m}, 1 \mathrm{~m})$ will converge to the origin $T(0,0)$, Obstacle is the orange point located 
at $(-0.3 m, 0.6 m)$. The dry and viscous friction parameters identified in previous research work [11] has been added to SimMechanics to be as close as possible to reality and in order to validate the performance and the robustness of our control laws. The two control laws described above have been implemented to be validated and to study the system behavior. The platform navigates from the initial position to the desired position and avoids the obstacle in both kinematic and dynamic controls as presented in Fig. 8 and Fig. 9.

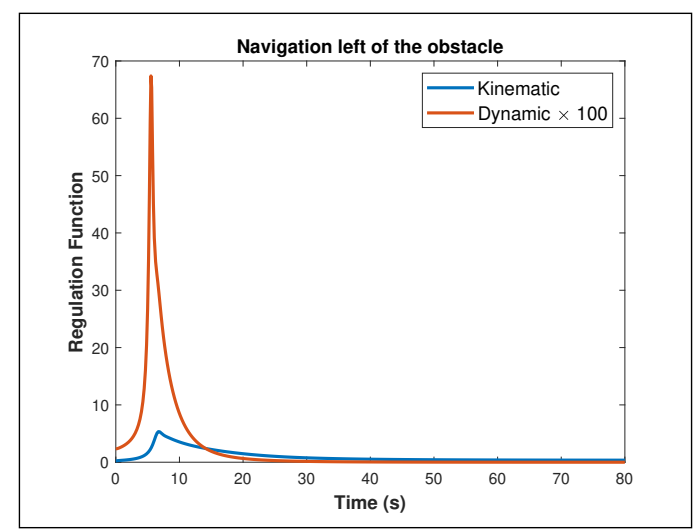

Fig. 10. Comparaison of regulation function $\xi$ in kinematic and dynamic

The results show that both control laws ensure convergence and collision avoidance. The trajectory from the dynamic control is smoother according to the regulation function Fig. 10 and the force/torque required is lower than those of the kinematic, while the convergence time is faster in kinematic control. Fig. 2 shows an image of the video showing the experimental application and validation of the proposed methods.

\section{Second scenario: Automatic mode}

First, a skiing trajectory was planned and defined on the virtualized mountain using Bezier polynomial interpolation:

$$
B(t)=\sum_{i=0}^{n}\left(\begin{array}{c}
n \\
i
\end{array}\right)(1-t)^{n-i} t^{i} P_{i} ; 0 \leq t \leq 1
$$

and it is shown in yellow in Fig. 11.

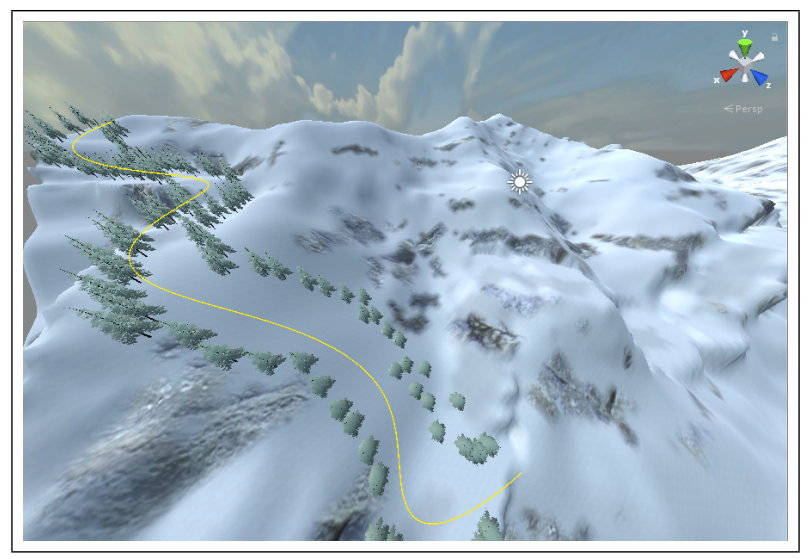

Fig. 11. Planned trajectory of Tréffléannaise track

All physics of downhill skiing describing forces acting on a skier such as downhill force, parallel downhill force, lateral force, centrifugal force, and kinematic friction force are taken into account using a UNITY rendering engine.

The scenario has unlimited or significant displacement (no constraint), while the robotic simulator (Evr@) has only $(1.5 m \times 1.5 m)$. To overcome the movement limitation, a common problem of all simulators, and to have continuous movement feeling and similar to the real ski scenario, a MCA also known as washout algorithm takes place [17] where these algorithms recover only the important information from reference acceleration based on the deep comprehension of the gravito-inertial organs of the ear (otoliths captures the linear acceleration while semicircular canals capture the angular velocity). Several MCA approaches have been used in this context, such as classic [18], adaptive [19] and optimal [20] approaches. The MCA can be summarized as follows:

- The HF (High Frequency) components of the accelerations are reproduced directly by a linear movement of the robotic platform.

- The LF (Low Frequency) components are restored by tilting the cabin of the robotic platform. This inclination will be interpreted as a linear acceleration if it is lower than the perception threshold not to be detected as a rotation. It is called "Tilt-ordination".

- The acceleration is zero. During this phase, the platform is returned to its neutral position. It is called "Washout".

To validate the chain operation from the virtual scenario to the real platform, a trajectory consisting of acceleration and deceleration phases will be rendered and applied. The trajectory is along the $\mathrm{X}$-direction and it is followed by both parallel robots and two sliding joints simultaneously. In Fig. 12 continuous lines represent the reference and

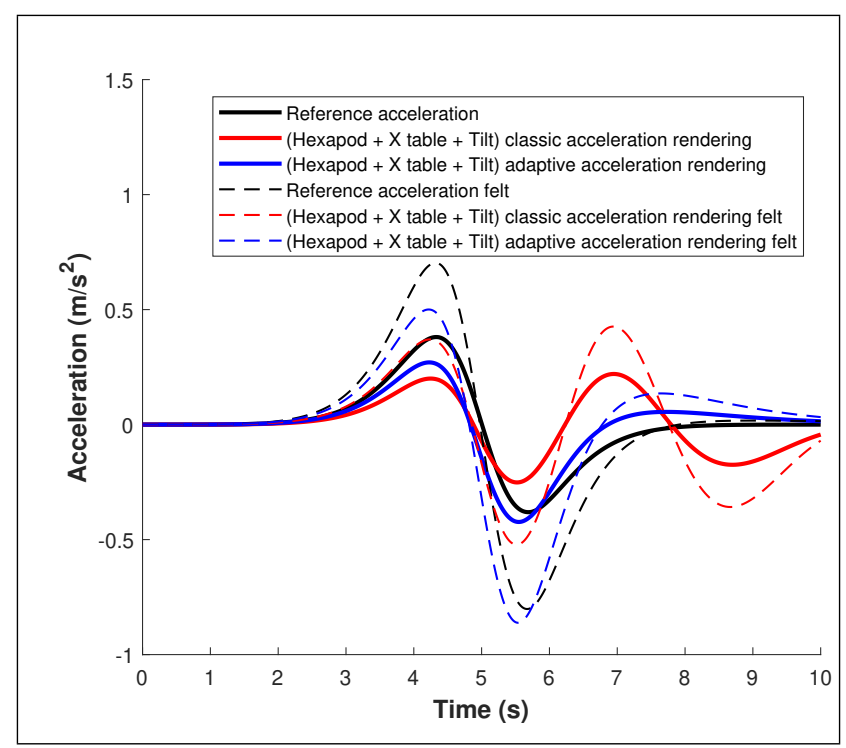

Fig. 12. Reference vs classic rendered acceleration

rendered accelerations and the dashed lines represent the accelerations felt after applying the human vestibular model presented in [21]. The filter's parameters of classic and adaptative approaches were manually adjusted according 
to displacement, speed, and acceleration capability of the robotic structure. As experimental validation, the desired achievable displacement coming from rendered acceleration was tracked by the real Evr@ simulator as it is shown in Fig. 13. The latency between theoretical and experimental trajectories is due to the time processing of the virtual scene, the dynamic modeling, and the hardware delay. The adaptive approach has a high deviation and latency compared to the classic approach due to the large computation of differential equations in real-time.

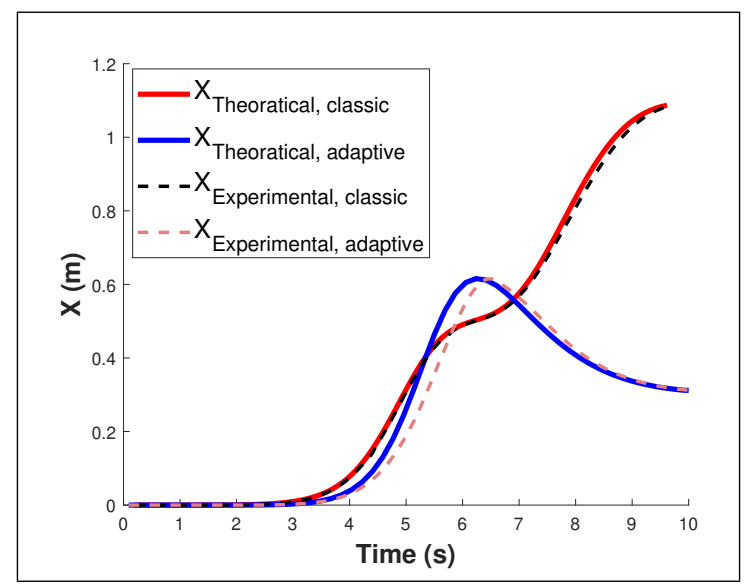

Fig. 13. Theoretical vs experimental displacement for classic and adaptive approach

\section{Conclusion And Perspective}

In this paper, we presented the construction of a mixed reality ski environment adapted to disabled people, we presented the mechatronic design of a series-parallel robot architecture platform. All mechanical and electronic parts are built and mounted from scratch in our laboratory. Our ambition is to reach the goal to have a better immersive platform for sliding movement simulation in both scenarios.

Towards our objective, to make human interaction more immersive in the virtual scene. In the first scenario, we have developed a control law allowing the avoidance of virtual obstacles when the user is considered active. For the second scenario, we go further in our goal firstly, by adding two series axes to the parallel robotic structure increasing the capacities of the platform and secondly, by applying appropriate algorithms such as the classic or the adaptive algorithms represented in Fig. 13.

The difference between desired and experimental trajectories are small, actually, we have the best results but they still require optimization. However, we are very confident about the platform behavior to make human interaction more immersive.

The future work and for security reasons will be concentrated on the investigation and the use of the humanoid robot NAO and after that, it permits us to utilize our robotic platform for human being interaction. Cold air devices and haptic force feedback can be added to increase human immersion. In the end, we study in-depth the human factors and ergonomic challenges.

\section{REFERENCES}

[1] World Health Organization paper in Apr 04, 2019. Available at: https://www.worldbank.org/en/topic/disability

[2] Ku, Jeonghun, Jang, Dongpyo , Ahn, Heebum , Lee, Jaemin , Kim, Jeong, Lee, Bumseok, Kim, In, Kim, Sun. (2002). The development and clinical trial of a Driving Simulator for the handicapped. Studies in health technology and informatics. 85. 240-6. 10.3233/978-1-60750929-5-240.

[3] Pierre Boudoin, Christophe Domingues, Samir Otmane, Nassima Ouramdane, and Malik Mallem. Towards multimodal human-robot interaction in large scale virtual environment. In in the 3rd ACM/IEEE International Conference on Human Robot Interaction (HRI), pages 359-366. ACM,IEEE, 12-15 mars 2008.

[4] G. Bouyer, A. Chellali and A. Lécuyer, "Inducing self-motion sensations in driving simulators using force-feedback and haptic motion," 2017 IEEE Virtual Reality (VR), Los Angeles, CA, 2017, pp. 84-90. doi: 10.1109/VR.2017.7892234

[5] Parallel Robots, J.-P Merlet - Kluwer Academic Publishers - 2006

[6] Mercedes-Benz "moving-base" driving simulator in Sindelfingen. Retrieved from: https://www.mercedes-benz.com/en/innovation/vehicledevelopment/the-mercedes-benz-driving-simulation-center/.

[7] Pan, Weidong and Yiannis E. Papelis. "Real-time dynamic simulation of vehicles with electronic stability control : Modeling and validation." (2005).

[8] Ali Amouri, Fakhr-Eddine Ababsa. Sliding Movement Platform for Mixed Reality Application. 7th IFAC Symposium on Mechatronic Systems, Sep 2016, Loughborough, United Kingdom. 49 (21), pp.662-667,.

[9] Dual-Ski model available at: http://www.handicat.com/at-num26894.html

[10] Hofmann K, Ohlsson M, Höök M, Danvind J, Kersting UG (2016) The influence of sitting posture on mechanics and metabolic energy requirements during sit-skiing: a case report. Sports Eng 19:213-218

[11] Houda T., Amouri A., Beji L., Mallem M. (2019) Dynamic Parameters Optimization and Identification of a Parallel Robot. In: Kecskeméthy A., Geu Flores F. (eds) Multibody Dynamics 2019. ECCOMAS 2019. Computational Methods in Applied Sciences, vol 53. Springer, Cham

[12] Ouarda Ibrahim, Wisama Khalil, Inverse and direct dynamic models of hybrid robots, Mechanism and Machine Theory, Volume 45, Issue 4, 2010, Pages 627-640, ISSN 0094-114X, https://doi.org/10.1016/j.mechmachtheory.2009.11.007.

[13] Khalil W., Dombre E., "Modeling, identification and control of robots", Hermès Penton, London, 2002.

[14] M. A. El Kamel, L. Beji and A. Abichou, "Nonholonomic mobile robots cooperative control for target capturing," 2008 Annual IEEE India Conference, Kanpur, 2008, pp. 548-552. doi: 10.1109/INDCON.2008.4768783

[15] Stabilization and regulation of mobile robots operating in groups thesis of Mohamed Anouar El Kamel - these.fr

[16] Description of Simscape Multibody available on: https://fr.mathworks.com/products/simmechanics.html

[17] L. Nehaoua, H. Arioui, S. Espie and H. Mohellebi, "Motion cueing algorithms for small driving simulator," Proceedings 2006 IEEE International Conference on Robotics and Automation, 2006. ICRA 2006., Orlando, FL, 2006, pp. 3189-3194. doi: 10.1109/ROBOT.2006.1642187

[18] M. Baseggio, A. Beghi, M. Bruschetta, F. Maran and D. Minen, "An MPC approach to the design of motion cueing algorithms for driving simulators," 2011 14th International IEEE Conference on Intelligent Transportation Systems (ITSC), Washington, DC, 2011, pp. 692-697. doi: 10.1109/ITSC.2011.6083053

[19] Dagdelen, Mehmet, Reymond, Gilles, Kemeny, Andras, Bordier, Marc, Maïzi, Nadia. (2009). Model-based predictive motion cueing strategy for vehicle driving simulators. Control Engineering Practice. 17. 9951003. 10.1016/j.conengprac.2009.03.002.

[20] H. Asadi, S. Mohamed, C. P. Lim and S. Nahavandi, "Robust Optimal Motion Cueing Algorithm Based on the Linear Quadratic Regulator Method and a Genetic Algorithm," in IEEE Transactions on Systems, Man, and Cybernetics: Systems, vol. 47, no. 2, pp. 238-254, Feb. 2017. doi: 10.1109/TSMC.2016.2523906

[21] Asadi, Houshyar; Mohamed, Shady ; Lim, Chee ; Nahavandi, Saeid. (2016). Robust Optimal Motion Cueing Algorithm Based on the Linear Quadratic Regulator Method and a Genetic Algorithm. IEEE Transactions on Systems, Man, and Cybernetics: Systems. 47. 1-17. 10.1109/TSMC.2016.2523906. 